\title{
Dominantly inherited distal nemaline/cap myopathy caused by a large deletion in the nebulin gene
}

\author{
Kirsi J. Kiiski ${ }^{\text {a,1 }}$, Vilma-Lotta Lehtokari ${ }^{a, 1, *}$, Anna K. Vihola ${ }^{\text {, Jenni M. Laitila }}{ }^{\text {a }}$, \\ Sanna Huovinen ${ }^{b}$, Lydia J. Sagath ${ }^{\mathrm{a}}$, Anni E. Eviläa, Anders E. Paetau ${ }^{\mathrm{c}}$, Caroline A. Sewry ${ }^{\mathrm{d}}$, \\ Peter B. Hackman ${ }^{\mathrm{a}}$, Katarina B. Pelin ${ }^{\mathrm{a}, \mathrm{e}}$, Carina Wallgren-Pettersson ${ }^{\mathrm{a}}$, Bjarne Udd ${ }^{\mathrm{a}, \mathrm{f}, \mathrm{g}}$ \\ ${ }^{a}$ The Folkhälsan Research Center, Helsinki, Finland and Medicum, University of Helsinki, Helsinki, Finland \\ ${ }^{\mathrm{b}}$ Department of Pathology, Tampere University Hospital, Fimlab Laboratories, Tampere, Finland \\ ${ }^{\mathrm{c}}$ Department of Pathology, HUSLAB, Helsinki University Hospital \& University of Helsinki, Finland \\ ${ }^{\mathrm{d}}$ Dubowitz Neuromuscular Centre, UCL Institute of Child Health and Great Ormond Street Hospital, London, UK \\ ${ }^{\mathrm{e}}$ Molecular and Integrative Biosciences Research Programme, Faculty of Biological and Environmental Sciences, University of Helsinki, Helsinki, Finland \\ ${ }^{\mathrm{f}}$ Neuromuscular Research Center, Tampere University and University Hospital, Tampere, Finland \\ ${ }^{\mathrm{g}}$ Department of Neurology, Vaasa Central Hospital, Vaasa, Finland
}

Received 2 July 2018; received in revised form 29 November 2018; accepted 16 December 2018

\begin{abstract}
We report the first family with a dominantly inherited mutation of the nebulin gene $(N E B)$. This $\sim 100 \mathrm{~kb}$ in-frame deletion encompasses $N E B$ exons 14-89, causing distal nemaline/cap myopathy in a three-generation family. It is the largest deletion characterized in $N E B$ hitherto. The mutated allele was shown to be expressed at the mRNA level and furthermore, for the first time, a deletion was shown to cause the production of a smaller mutant nebulin protein. Thus, we suggest that this novel mutant nebulin protein has a dominant-negative effect, explaining the first documented dominant inheritance of nebulin-caused myopathy. The index patient, a young man, was more severely affected than his mother and grandmother. His first symptom was foot drop at the age of three, followed by distal muscle atrophy, slight hypomimia, high-arched palate, and weakness of the neck and elbow flexors, hands, tibialis anterior and toe extensors. Muscle biopsies showed myopathic features with type 1 fibre predominance in the index patient and nemaline bodies and cap-like structures in biopsies from his mother and grandmother. The muscle biopsy findings constitute a further example of nemaline bodies and cap-like structures being part of the same spectrum of pathological changes.
\end{abstract}

(C) 2019 Elsevier B.V. All rights reserved.

Keywords: Dominant nemaline myopathy; Distal myopathy; Cap myopathy; Nebulin; Nemaline myopathy-CGH Array.

\section{Introduction}

Nemaline myopathy (NM) is one of the most common of the congenital myopathies, which include clinically, histologically and genetically variable neuromuscular disorders defined on the basis of structural abnormalities in the muscle fibres [1]. The diagnostic hallmark is nemaline (rod) bodies, stained red with the Gömöri trichrome technique at light microscopy and appearing as dark lattice structures

\footnotetext{
* Corresponding author.

E-mail address: vilma.lehtokari@helsinki.fi (V.-L. Lehtokari).

${ }^{1}$ These authors contributed equally to this work.
}

with electron microscopy [2,3]. In nemaline myopathy, muscle weakness of the proximal muscles usually dominates the clinical picture, later accompanied by a distal component, while severity varies from very mild to very severe. Cap myopathy belongs to the spectrum of nemaline myopathies, as cap-like areas are often seen together with nemaline rods in the same patient or family. Caps are focal, peripheral areas with disrupted myofibrils and thickened $\mathrm{Z}$ lines or small rod-like structures $[4,5]$. Distal forms of nemaline myopathy have been reported in four families [6-8].

Mutations in eleven different genes are known to cause nemaline myopathy. Eight of these genes, alpha-tropomyosin (TPM3) (MIM: 191030) [9], nebulin (NEB) (MIM: 161650) 
[10], skeletal alpha-actin (ACTA1) (MIM: 102610) [11], troponin T1 (TNNT1) (MIM: 191041) [12], beta-tropomyosin (TPM2) (MIM: 190990) [13], cofilin 2 (CFL2) (MIM: 601443) [14], leiomodin-3 (LMOD3) (MIM: 616112) [15], and myopalladin (MYPN) (MIM: 608517) [16] genes encode structural components of the muscle sarcomere thin filament. The other three genes, Kelch repeat- and btb/poz domain-containing protein 13 (KBTBD13) (MIM: 613727) [17], Kelch-like gene family member 40 (KLHL40) (MIM: 615340) [18], Kelch-like gene family member 41 (KLHL41) (MIM: 607701) [19] genes encode proteins involved in the stability or turnover of the thin filament. Mutations in TPM2, TPM3, ACTA1 and MYPN have been reported to cause cap myopathy or cap-like structures in addition to nemaline rods [5,20-24]. The mode of inheritance of the nemaline myopathies may be dominant or recessive, or the disorder may be caused by a de novo dominant mutation.

The vast majority of patients with $N E B$ mutations have been diagnosed with nemaline myopathy. However, $N E B$ mutations have also been identified in patients with distal nebulin myopathy (four families) [25], distal nemaline myopathy (three families) [6], core-rod myopathy (one family) [26], distal core-rod myopathy (one family) [27] and fetal akinesia/lethal multiple pterygium syndrome (four families) $[7,28]$. Thus, the $N E B$-caused myopathies characterized to date constitute a clinically, histologically and genetically heterogeneous group of muscle disorders.

Distal myopathies, likewise showing a spectrum of histopathological findings and clinical phenotypes, have varying ages of onset of symptoms from early childhood to late adulthood, and varying patterns of muscle involvement $[29,30]$. Several genes have been identified as causative of distal myopathies, some of which cause other myopathy phenotypes also, and the mode of inheritance is either autosomal recessive or dominant depending on the causative gene $[29,30]$.

Nebulin is one of the largest proteins in vertebrates (600-900 kDa), encoded by the $249 \mathrm{~kb} N E B$ gene including 183 exons with a theoretical full-length cDNA sequence of $2.6 \mathrm{~kb}$ (Ref.Seq. NM_001271208). Nebulin is expressed mainly in striated muscle, where it is essential for stabilising the thin (actin) filament of the muscle sarcomere as well as for the proper functioning of muscle contraction [31,32]. The extensive alternative splicing of $N E B$ creates a high number of different nebulin isoforms [33]. Nebulin is a highly conserved protein consisting of simple and super repeats. The middle part of nebulin contains a triplicate region in which eight exons are repeated three times almost identically [31-33].

To date, we have identified more than 240 different disease-causing variants in $N E B$ in more than 210 families $[7,34,35]$. The majority are unique, or present in only a few families. Of the affected families, $80 \%$ carry compound heterozygous and $20 \%$ homozygous $N E B$ mutations $[7,34,35]$. Of all the different $N E B$ mutations, $95 \%$ consist of point mutations and small indels, whereas different large copy number variations $(\mathrm{CNV})$ are rare, accounting for only 5\% of the mutations $[7,35]$. However, copy number variation of the $N E B$ triplicate region was shown to be recurrent and present in $14 \%$ in a cohort of 270 families studied using the nemaline myopathy-comparative genomic hybridisation array (NM-CGH Array). NEB triplicate region $\mathrm{CNV}$ was interpreted to be disease-causing in $4 \%$ of the families [35]. In addition to the well-described $N E B$ exon 55 deletion and the recurrent $N E B$ triplicate region $\mathrm{CNV}$, we have identified 11 different pathogenic large duplications and deletions in $N E B$. Of the 210 families with one or both pathogenic $N E B$ variations identified, $17 \%$ carry a large pathogenic $\mathrm{CNV}$ (unpublished data). All mutations characterized in $N E B$ thus far have shown recessive inheritance [7,35]. Of the 240 different $N E B$ mutations, $90 \%$ have been identified as compound heterozygous and $7 \%$ both as homozygous and compound heterozygous, while $3 \%$ of the mutations were identified as homozygous only.

Most of the $N E B$ mutations identified in the ten published families with distal nebulin or distal nemaline myopathy have also been identified in nemaline myopathy and/or core-rod myopathy patients $[6,7,25,27]$. Hitherto, large CNVs in $N E B$ have been identified only in patients with recessive nemaline myopathy.

Here, we present the first description of a dominantly inherited $N E B$ mutation. This novel $\sim 100 \mathrm{~kb}$ in-frame deletion was found to cause a distal form of nemaline/cap myopathy in a three-generation Finnish family.

\section{Materials and methods}

\subsection{Patients}

The patients enrolled in the study were the index patient (III-1), his mother (II-2) and maternal grandmother (I-3) (Fig. 1). The index patient (Fig. 2A-D) presented with muscle weakness at the age of 3 years, and subsequently his mother (Fig. 2E-F) and maternal grandmother were also found to be affected. Moreover, the healthy siblings of the mother and grandmother were clinically examined, with normal results, and the maternal uncle underwent muscle CT, with a normal result.

\subsection{Samples}

We received genomic DNA samples and muscle biopsies from the three patients (I-3, II-2 and III-1), and DNA samples of ten healthy first-degree relatives for segregation studies (Fig. 1). The study has been approved by the Ethics Committee of the Children's Hospital, and the Helsinki University Hospital, University of Helsinki, Helsinki, Finland.

\subsection{Molecular genetic studies}

In order to identify the causative mutation(s) in this family, mutations known to cause distal myopathies were screened in the patients (I-3, II-2, III-1), including known mutations in titin (TTN), myosin heavy chain 2 (MYH2), myosin heavy chain 7 (MYH7), myotilin (MYOT) and Z-disc 


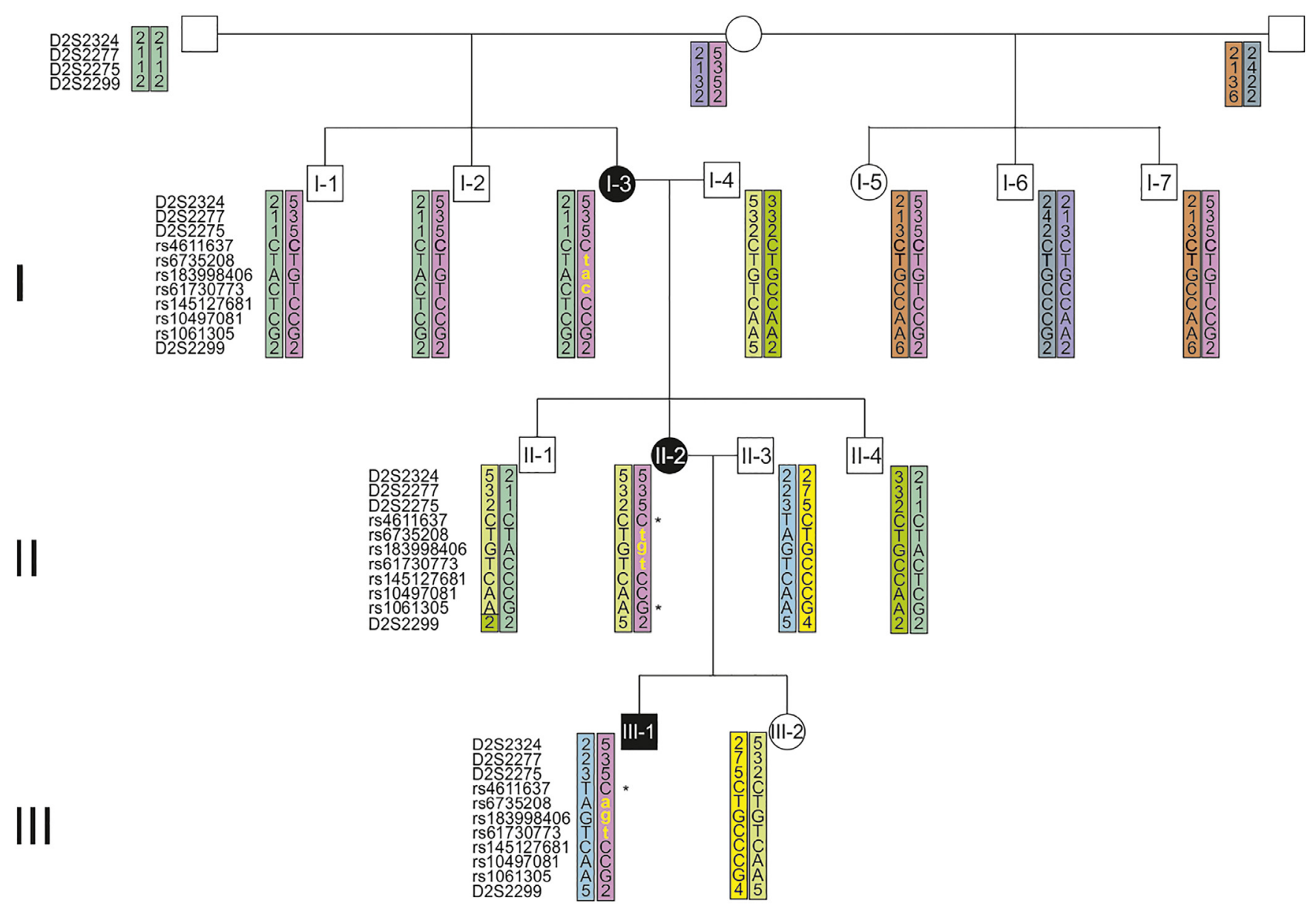

Fig 1. Family tree and the haplotypes. The family tree and the haplotypes of each individual genotyped in the NEB region in chromosome 2 . The microsatellite markers locate around $N E B$ and SNPs are within the gene region. The colours of the haplotypes visualize the inheritance patterns of the alleles. The deleted allele is presented in yellow font (I-3, II-2 and III-1). Asterisks indicate the SNPs analysed in the cDNA. The heterozygosity of the SNPs show that both ends of the $N E B$ mRNA are present in both alleles.

associated alternatively spliced gene encoding a PDZ-LIM domain-binding 3 protein (ZASP) genes. The DNA sample of the index patient (III-1) was checked by Sanger sequencing for the pathogenic $N E B$ variants hitherto identified in the Finnish population (NEB exons 8, 32, 50, 54, 61, 65, 68, $75,117,122,134,136,142,151,163,173$ and 182). These Finnish $N E B$ variants are listed in the LOVD database and in our previous publications $[7,10,35,36]$.

Whole-genome linkage analyses were performed at deCode (Iceland). The $N E B$ haplotypes for the family were constructed using single nucleotide polymorphisms (SNPs) in the exons $4,10,31,65,122,151,173$ and 181 for all the 13 family members from whom DNA samples were available (three affected and 10 unaffected family members) (Fig. 1).

The Array Comparative Genomic Hybridization Method (Oxford Gene Technology IP Limited, Oxford, UK) was used for targeting the known nemaline myopathy genes with a high-density tiling approach in our custom NM-CGH $8 \times 60 \mathrm{k}$ Microarray. In addition, the CytoSure ISCA+SNP $4 \times 180 \mathrm{k}$ array was used to analyse copy number variations of the whole-genome. The labelling, hybridisation and scanning was done according to the manufacturer's protocol and data analysis and interpretation was done using CytoSure Interpret Software v.4.9. (GRCh37) as previously described [37]. The CBS algorithm was used with segmentation-based normalisation and specific settings created for the GC-rich probes for the NM-CGH Array analysis. All 13 family members were analysed using the NM-CGH Array.

Multiplex ligation-dependent probe amplification (MLPA) was performed using the MLPA SALSA kit according to the manufacturer's protocol (MRC-Holland, Amsterdam, The Netherlands), using self-designed oligonucleotide probes to target the following regions of $N E B$ : the promoter region and exons 14, 24, 52, 55, 86, 87, 89, 106, 171, 180, 183 (detailed probe information available on request). MLPA was performed and analysed as previously described [37]. All three affected family members and seven unaffected family members were analysed using MLPA.

Whole-exome sequencing (WES) was performed with two different approaches. The first WES was done using Axeq Korea/USA (Illumina TruSeq) of one affected (II-2) and one unaffected family member, a maternal uncle (II-1). The second WES method was done on the index patient (III-1) using Agilent SureSelectXT All Exon 50Mb target enrichment 


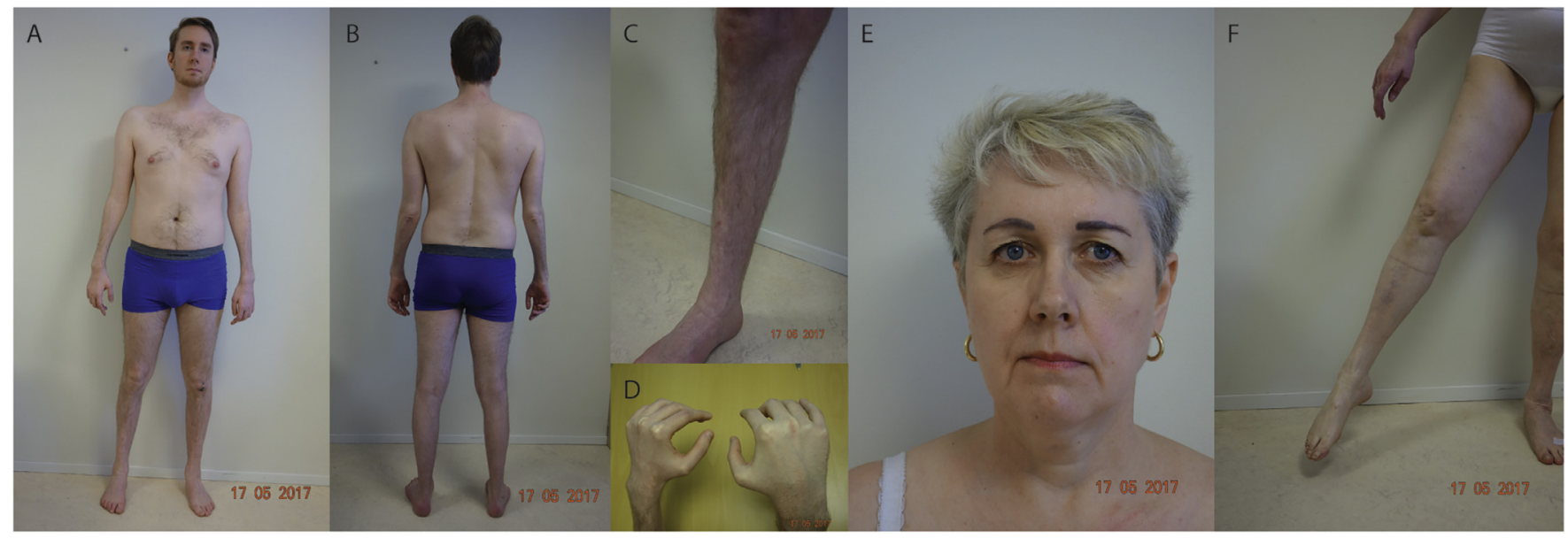

Fig. 2. The index patient and his mother. (A-C) The index case (III-1) with mainly distal muscle atrophy and weakness of the wrist and finger extensors as shown in D. (E) The mother of the index case with slight facial hypomimia and (F) muscle atrophy of the lower legs with weakness of ankle dorsiflexion.

kit (protocol v1.2; Agilent Technologies, Santa Clara, CA, USA) on an Illumina HiSeq2000 platform using TruSeq v3 chemistry (Illumina Inc, San Diego, CA, USA) and analysis was completed using the Oxford Gene Technology exome sequencing pipeline.

Conventional karyotyping of the mother (II-2) was performed in blood lymphocytes using G-banding. All $183 N E B$ exons and exon-intron boundaries were Sanger sequenced and RT-PCR of $N E B$ was performed using mRNA from the muscle biopsy samples of the index patient (III-1) and his mother. Moreover, the variants identified in $M Y H 2$, $M Y B P C 1$ and DYS were sought for by sequencing in all 13 family members.

All primer sequences and PCR details are available from the corresponding author.

\subsection{Western blot analysis}

Frozen muscle sections (20 sections, thickness $12 \mu \mathrm{m}$ ) were prepared in pre-chilled Eppendorf tubes. To prepare crude myofibrillar fractions, ice-cold TE-sucrose (Tris-EDTA, pH 7.4, with 0.25 M sucrose and Halt Protease Inhibitor Mix, (Thermo Scientific) was added, following by vortexing full speed for $60 \mathrm{~s}$. The mixture was centrifuged $1,000 \times \mathrm{g}$ for $15 \mathrm{~min}$. The pellet was washed with TE-buffer and dissolved in WB sample buffer (Laemmli buffer with $20 \mathrm{mM}$ EDTA and $4 \mathrm{M}$ urea), mixed by vortexing and incubated at room temperature for $15 \mathrm{~min}$. Insoluble material was discarded after centrifugation, and the supernatant was used for SDS-PAGE. Equal amounts of the samples were run on standard 12\% SDS-PAGE gels and stained with Coomassie blue to assess the total protein content in each sample. Volumes were then adjusted to obtain equal protein loading. Ready-cast 7.5\% TGX gels (Bio-Rad Laboratories, CA, USA) were used to separate the nebulin protein by running $18 \mathrm{~h}$ at $50 \mathrm{~V}$. The proteins were transferred onto PVDF membranes using Bio-Rad TransBlot Turbo blotting device, Standard SD program $(25 \mathrm{~V}), 40 \mathrm{~min}$. The filters were incubated with primary antibody, clone $N E B$ 9C4 B5E5 (provided by Glenn Morris, Oswestry), at $1: 300$ dilution at $+8{ }^{\circ} \mathrm{C}$ for $18 \mathrm{~h}$, with gentle agitation, and detected using ECL. ChemiDoc reader and ImageLab software (Bio-Rad Laboratories) were used to obtain images and for calculating the molecular weights of the bands of unknown size.

\subsection{Immunolabelling of muscle sections}

Immunolabelling was performed on $10 \mu \mathrm{m}$ sections of muscle frozen in isopentane cooled in liquid nitrogen. Sections were immunolabelled with primary antibodies against $\mathrm{N}$-terminal nebulin (rabbit polyclonal $\mathrm{H}-300$, diluted $1: 10$ in $1 \%$ bovine serum albumin, BSA; SC28286, Santa Cruz, Dallas, TX, USA), and nebulin super repeat S21 (mouse monoclonal 9C4 B5E5, diluted 1:100; provided by Glenn Morris, Oswestry).

Alexa Fluor Donkey anti-Rabbit 488 (1:500 in 1\% BSA; A21206, Thermo Fisher Scientific Waltham, MA, USA) was used as the secondary antibody for the N-terminal nebulin H-300-ab, and Alexa Fluor Goat anti-Mouse 546 (1:500 in 1\% BSA; A11003, Thermo Fisher Scientific) for the S21-mAb.

The sections were fixed in 4\% PFA (paraformaldehyde) for 10 minutes, permeabilised in $0.2 \%$ Triton X-100 for 10 minutes and blocked in 5\% BSA for 30 minutes before adding the primary antibody (at $\left.+4{ }^{\circ} \mathrm{Co} / \mathrm{n}\right)$. The secondary antibody was allowed to bind for $1 \mathrm{~h}$ at RT. Hoechst (1:5000 in PBS, Thermo Fisher Scientific) was added to counterstain nuclei before mounting in Fluoromount (Sigma-Aldrich, St. Louis, MO, USA). Optical sections of $0.9 \mu \mathrm{m}$ were imaged using a ZEISS LSM 880 confocal microscope.

\section{Results}

\subsection{Clinical findings}

The index patient (III-1) was the first child of non-consanguineous Finnish parents. His neonatal period and 

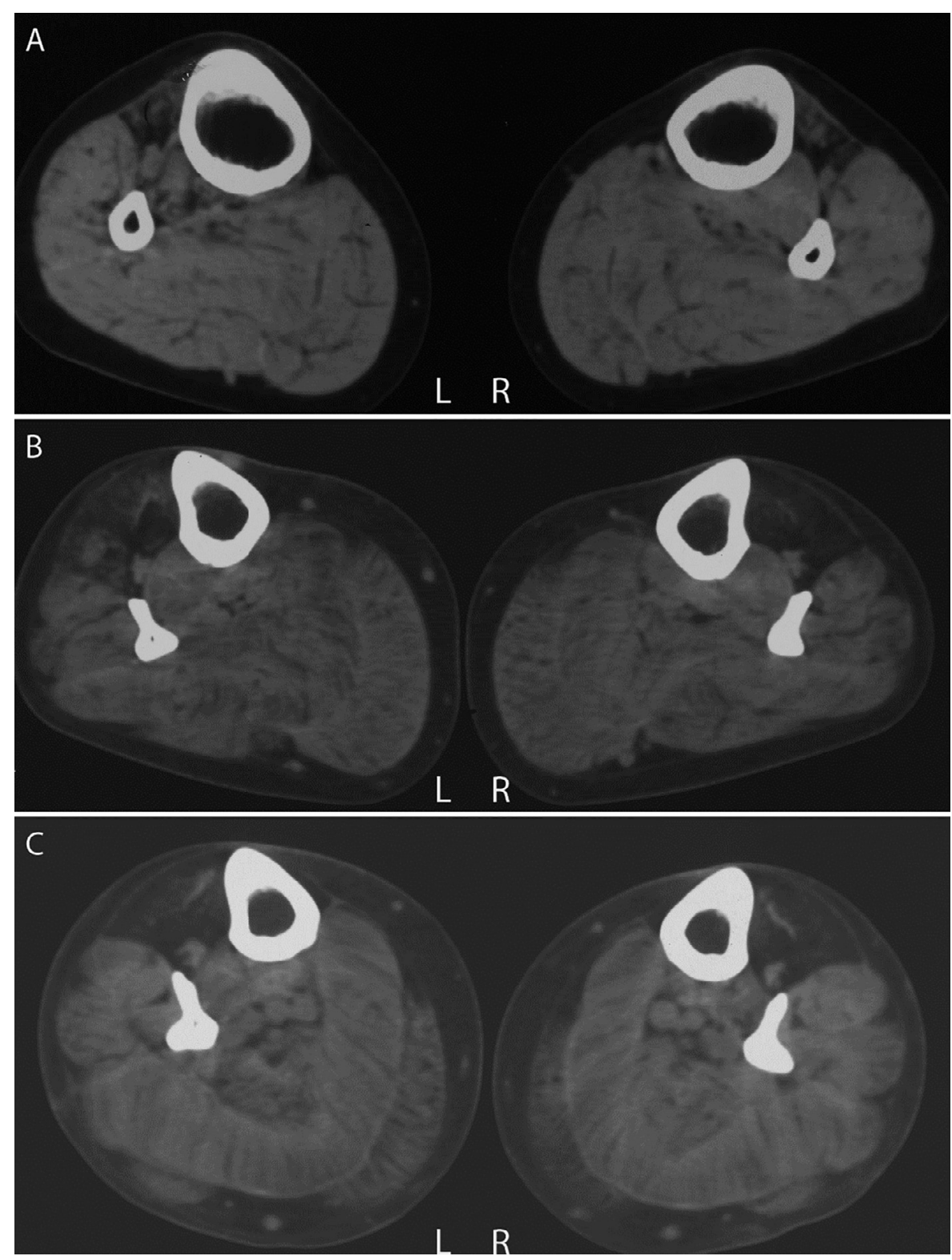

Fig. 3. CT scans of lower leg muscles. (A) The index case (III-1) at the age of 12 years shows complete fatty degeneration of all anterior compartment muscles and minor diffuse degenerative change in posterior calf muscles. (B) The mother (II-2) at the age of 45 years shows complete fatty degeneration of all anterior compartment muscles and minor diffuse degenerative change in the soleus muscles. (C) The grandmother (I-3) at the age of 64 years shows complete fatty degeneration of all anterior compartment muscles. The other muscles of the lower legs are normal for the age.

infancy were medically unremarkable. At the age of three, he was noted to have foot drop, which led to investigations at the Children's Hospital, University of Helsinki at the age of 4.5 years. On examination, he was found to have an elongated face. He was unable to walk on his heels, hopping on one leg was difficult and the extensors of the big toes were weak. The strength in other muscle groups was normal. Electromyography showed small motor unit potentials and neurography showed normal conduction velocities. Serum concentrations of creatine kinase were normal (116 IU/l, normal range 20-220 IU/l). A tentative diagnosis of tibial muscular dystrophy (TMD) was made, the early age of onset and the facial weakness however making this diagnosis unlikely. CT scans of the lower leg muscles at the age of 10 years showed extensive atrophy and fatty degeneration of all anterior compartment muscles (Fig. 3A).

Since then, the course of the disease has been slowly progressive. The young man, now aged 30 years, reported slight difficulties doing push-ups and rising from the squatting position, grip strength having become weaker, and carrying heavy loads more difficult. He was able to climb stairs, albeit a bit slowly.

On examination at the age of 30 years, he was a tall, slender man with moderate atrophy of the distal muscles of the upper and lower limbs. Atrophy was especially evident in the anterior lower legs, thenar, hypothenar and EDB 
muscles, with asymmetry of the calf muscles, the right being thinner (Fig. 2A-B). He had a slight hypomimia and clearly high-arched palate. Both ankles had $90^{\circ}$ contractures and the feet were high-arched (Fig. 2C). On muscle testing, the neck flexors and the finger extensors (Fig. 2D) were especially weak, 2/5 on the MRC scale, while toe and finger flexors had a strength of 4 . He was unable to walk on his heels, hopping on one leg was more difficult on the right, and the ankle dorsiflexors as well as the toe extensors showed a weakness of 0 . The shoulder girdle muscles and elbow flexors had a power of 4, while all other muscles showed normal strength.

The mother (II-2) of the index patient was doing well in terms of her muscle strength and had not sought medical attention because of any weakness noted. She had, however, undergone surgery for a benign brain tumour, the operation and the post operative immobility leaving her with asymmetric muscle weakness and slight difficulties keeping her balance. Subsequently, she had not noted any additional, clear deterioration in muscle strength.

As a child, by history, she had good physical endurance, although not as good as the best of her peers, and she participated in long-distance cross-country skiing competitions. She reported always having had a slight clumsiness. Examinations initiated at the age of 35 years because of the tentative diagnosis of TMD in her son showed marked weakness of ankle dorsiflexion, and on muscle CT scans, fatty degeneration of the anterior compartment muscles of the lower legs (Fig. 3B).

On examination at the age of 57 years, she had a normal build, with slight atrophy of the distal muscles of the upper and lower limbs, including the wrist flexors and extensors, all hand muscles, especially the thenar muscles and the interossei, and the anterior compartment of the lower legs (Fig. 2E). She had a mild scoliosis and low arches of the feet. She had slight hypomimia (Fig. 2F) and somewhat high-arched palate. She was unable to walk on her heels and had difficulty getting up from the squatting position. Muscle testing revealed a weakness of $0 / 5$ in the ankle dorsiflexors and the toe extensors, of 4 or $4+$ in the neck flexors, the shoulder girdle, the extensors and flexors of the wrists and the flexors of the fingers, while the finger extensors were clearly weaker (4-). The axial muscles showed only very slight weakness (5-), while the strength of the knee flexors and extensors, the ankle and the toe plantar flexors was normal. Serum concentrations of creatine kinase were normal.

The maternal grandmother (I-3) of the index patient was first examined at the age of 60 years as part of the family study. She had not previously sought medical attention for any muscle weakness. She had, however, had foot drop as long as she could remember, and had been unable to stand on her heels since the age of 10 years. CT scans of the lower leg muscles showed extensive fatty degeneration in all anterior compartment muscles of the lower legs (Fig. 3C). When examined at the age of 73 years, she had weakness of $2 / 5$ in the ankle dorsiflexors, 4 in the neck flexors and 5- in the proximal parts of the lower limbs. Serum concentrations of creatine kinase were normal.

\subsection{Muscle histopathology}

A muscle biopsy from the quadriceps femoris muscle of the index patient (III-1) at the age of 4 years showed marked fibre type 1 predominance. No specific structural abnormalities were identified in the muscle fibres, and Gömöri trichrome staining as well as toluidine blue-stained semithin sections failed to reveal any nemaline rods. The second biopsy from lower leg muscles taken in the context of surgery at the age of 12 years showed type 1 predominance and a biphasic distribution of type 1 fibre size, with fibre sizes ranging from 10 to $90 \mu \mathrm{m}$ in diameter (Fig. 4A). The third biopsy obtained from the gastrocnemius medialis muscle of III-1 at the age of 28 years showed end-stage fibrotic pathology and severe atrophy involving both fibre types. Occasional cytoplasmic bodies and thickened $\mathrm{Z}$ discs were observed on Gömöri trichrome staining. Typical nemaline rods were not observed in any of the biopsies.

Gastrocnemius medialis biopsies were obtained from the grandmother (I-3) at the age of 73 years and from the mother (II-2) at the ages of 45 and 55 years. Those from the mother revealed type 1 fibre predominance of more than $80 \%$. There was an excess of fibrous and fatty tissue, as well as atrophy involving both type 1 and 2 fibres. Fibres of both types showed numerous randomly distributed sarcoplasmic nemaline bodies and clusters of nemaline bodies also in peripheral cap-like areas (Fig. 4B-D). Subsarcolemmal pale-staining cap structures were also identified. In addition, some peripheral areas contained bright-red structures corresponding to clusters of nemaline rods. The histopathological findings in the biopsy of the grandmother (I-3) were similar, except that type 1 fibre predominance was not observed. On electron microscopy nemaline bodies and cap-like structures were identified in both patients' (I-3 and II-2) biopsies (Fig. 4E-F).

The confocal microscopy confirmed the presence of both ends of nebulin in muscle tissue of the index patient (Fig. 4G-I). No structural abnormalities or protein aggregates were detected with either of the nebulin antibodies.

\subsection{Genetic findings}

Using the NM-CGH Array a massive deletion removing the exons 14-89 of $N E B$ was detected (Fig. 5A). This deletes $\sim 99-106 \mathrm{~kb}$ of genomic DNA. The deletion covers exons $14-81 \sim 89-96 \mathrm{~kb}$, and one copy of the $N E B$ triplicate region (exons $82-89) \sim 10 \mathrm{~kb}$ in size.

The molecular karyotype of the three affected family members according to the HGVS nomenclature is; chr2:g.(152454645_152456955)_(152554712_152561404)del (GRCh37) relating to c. $\left(1257+1 \_1258-1\right) \_(13,788+$ 1_13,789-1)del.

The NM-CGH Array did not reveal the exact breakpoint residing in intron 13 because of a $6.7 \mathrm{~kb}$ gap between probes. The reason for the design not including probes in this particular region is that the sequence contains various $A l u$ and LINE1 sequence repeats, hindering the designing of 

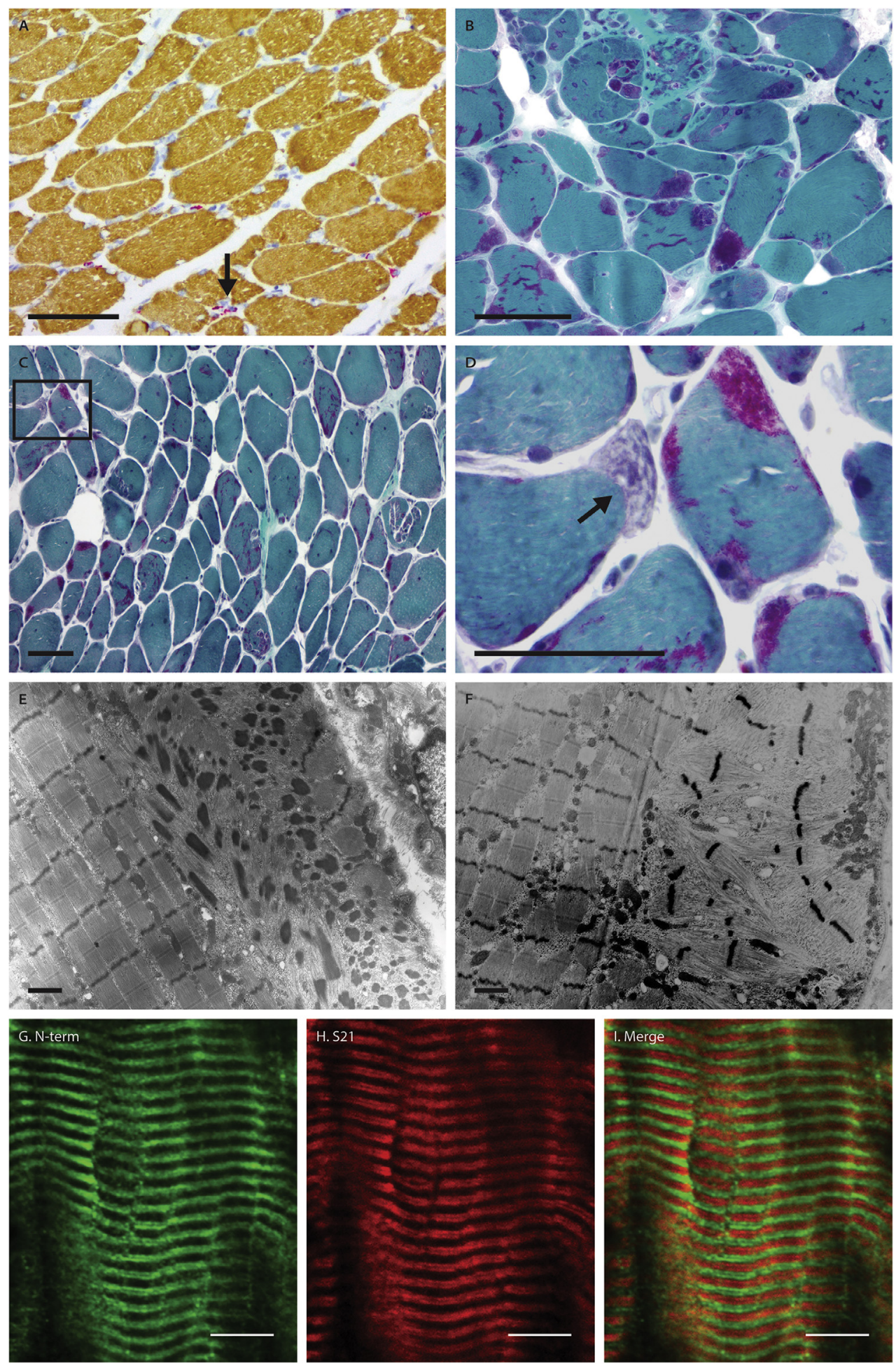

Fig. 4. Biopsy findings of the index patient (III-1). (A) Lower leg muscle biopsy of the index patient (III-1) at the age of 12 years double-stained with MyHC slow (brown) and MyHC fast (2A fibres red) demonstrates variation of fibre size and type 1 fibre predominance. (B-D) All type 2A fibres are very small (arrow). Gömöri trichrome stains of GM muscles of the grandmother (I-3) at the age of 73 years (B) and of the mother (II-2) at the age of 45 years (C, D) show red-staining nemaline rods in the intermyofbrillar spaces and clusters of nemaline rods in peripherally located cap-like structures. (D) Pale green or grey subsarcolemmal cap-like structures with disorganized myofibrillar structure are also present (arrow). (E) Electron microscopy of the GM muscle biopsy of II-2 shows a cluster of longitudinally and transversely oriented electron-dense nemaline rods located at the periphery of the fibre. (F) On the right side of the micrograph a subsarcolemmal cap structure with disorganized thin filaments and thickened Z discs is shown. Scale bar $=100 \mu \mathrm{m}(\mathrm{A}-\mathrm{D})$, scale bar $=1 \mu \mathrm{m}$ (E-F). (G-I) Immunofluorescence staining of muscle sections: Confocal microscopy of the nebulin N-terminus at the thin filament pointed end (G) and super repeat $21(\mathrm{~S} 21)(\mathrm{H})$, flanking the $\mathrm{Z}$ disc, in the index patient (III-1). Staining confirmed the expression of both ends of nebulin in correctly assembled sarcomeres (I). No protein aggregates were observed. The images show a single optical section of $0.9 \mu \mathrm{m}$. Scale bar $=5 \mu \mathrm{m}$. 

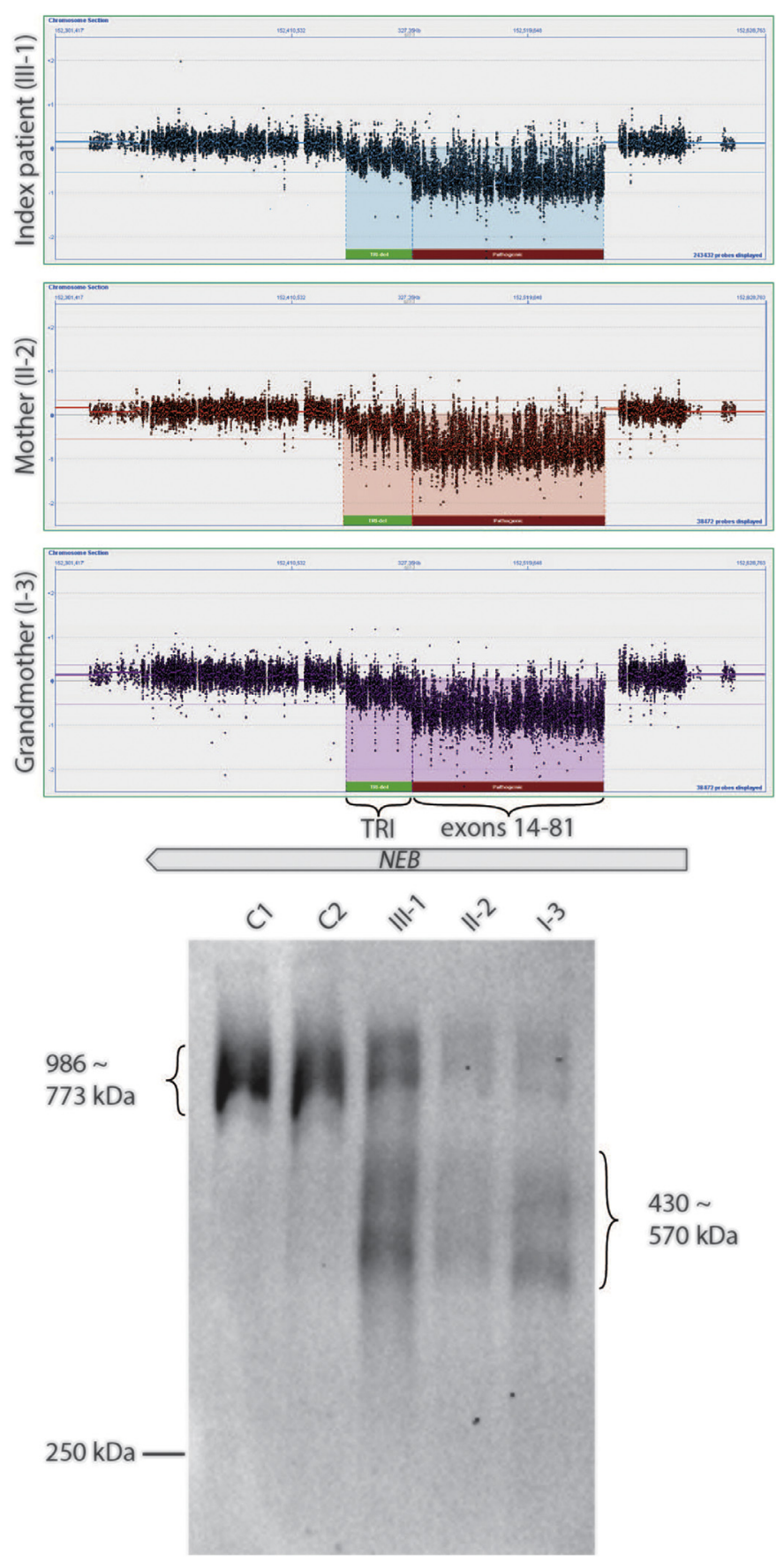

Fig. 5. (A) NM-CGH array and the Western blot profiles of the three affected family members. The array profiles of the index patient (III-1), the mother (II-2) and the grandmother (I-3) all show the same deletion of $N E B$ exons $14-81$ as well as a one-copy loss of the $N E B$ triplicate region (exons 82-89). (B) Nebulin Western blot analysis shows the corresponding short nebulin isoforms present in the affected family members. The full-size nebulin bands were designated as 986 and $773 \mathrm{kDa}$ (Uniprot P20929), and hence, the short nebulin isoforms were approximated as 430 570 kDa. C1, C2: controls.

unique probes. The other breakpoint is interpreted to reside in intron 89, leading to loss of one copy of the $N E B$ triplicate region. Thus, two copies of the triplicated region remain in the mutated allele, and the patients each have a total of $5 / 6$ copies. The same deletion pattern was found to be present in all three affected family members, indicating that the mutation constitutes one single deletion affecting one allele.

We analysed the DNA samples also from ten unaffected family members and they showed normal array profiles of $N E B$.

The deletion was verified using MLPA in all three affected family members. In addition, samples from seven unaffected relatives were studied, yielding normal results. We tried to reveal the exact breakpoints by sequencing the genomic DNA of the index patient (III-1). Despite several sequencing attempts, this could not be achieved. Based on our previous studies, we know that the repetitive triplicate region may cause major difficulty in Sanger sequencing.

Prior to doing the NM-CGH Array analysis, we excluded all known Finnish distal myopathy mutations and $N E B$ mutations in the index patient using Sanger sequencing (III-1). To locate the causative gene for this family, whole-genome linkage analysis was performed. The affected family members shared one haplotype spanning both $N E B$ and TTN on chromosome 2, which was compatible with linkage to a possible dominantly inherited disease. The first exome sequencing was performed on samples from the mother of the index patient (II-2), and one unaffected family member, a maternal uncle, II-1 (Fig. 1) (Illumina TruSeq). The most promising rare variants were analysed by Sanger sequencing in all affected family members. However, this did not reveal the causative gene. After that, the NM-CGH Array was performed, revealing the large deletion in $N E B$.

As only recessive mutations have been characterized in $N E B$ previously, we sequenced all 183 nebulin exons of the index patient (III-1) and his mother (II-2). We failed to find a second pathogenic variant in $N E B$. Subsequently, the entire $N E B$ cDNA of the mother was sequenced, excluding deep intronic mutations possibly disrupting the correct splicing of $N E B$. No additional mutations were detected. Studying muscle cDNA from the index patient and his mother, we found that heterozygous SNPs were present at the $5^{\prime}$ and $3^{\prime}$ ends of $N E B$, verifying the expression of both $N E B$ alleles (Fig 1). This gave further support to the hypothesis of dominant inheritance.

Haplotyping in all the 13 family members using genomic DNA showed homozygosity for the middle part of $N E B$ in the affected family members only. Furthermore, haplotypes of the grandmother (1-3) and her siblings revealed that the grandmother's myopathy was due to a de novo mutation.

We also did a second WES study of a DNA sample of the index patient (III-1), using the Agilent SureSelectXT All Exon $50 \mathrm{Mb}$ target enrichment kit, to see if any pathogenic variants in other genes could have been missed. We identified heterozygous missense variants in $M Y H 2$ (p.Ile1515Thr) and in MYBPCl (p.Val337Ile) in the index patient, as well as in the healthy father (II-3), but neither of them in the affected mother (II-2). Moreover, a variant, c.957G $>$ T: p.Trp319Cys, was identified in heterozygous form in the $\mathrm{X}$-chromosomal dystrophin gene in the index patient, the mother, the grandmother (I-3) and also in the unaffected 
maternal uncle (II-1). As this variant was identified in the unaffected uncle, we considered it to be non-pathogenic.

We studied the DNA sample of the index patient (III-1) also using a whole-genome $4 \times 180 \mathrm{k}$ ISCA+SNP array. This did not reveal any other CNVs.

Furthermore, we analysed the karyotype of the mother (II-2) in blood lymphocytes to exclude any chromosomal rearrangements, such as balanced translocations. Her karyotype was normal.

To assess the expression of the mutant allele at the protein level, a Western blot analysis was performed of myofibrillar protein lysates extracted from the muscles of all three affected family members, and from healthy controls. This analysis showed that in addition to the normal-sized (approximately $800 \mathrm{kDa}$ ) nebulin protein, there were two smaller mutant proteins present in the samples from the affected family members that were not present in the control samples (Fig. 5B). The calculated size of the mutant nebulin is $470 \mathrm{kDa}$. This provides evidence that the mutant allele produces smaller-sized nebulin proteins, which are not degraded and show stable expression levels.

\section{Discussion}

We present here the first dominantly inherited $N E B$ mutation as the cause of a mild distal myopathy presenting in childhood with foot drop. Because of their mild symptoms, the mother (II-2) and grandmother (I-3) in the family were only examined after the clinical diagnosis in the index patient (III-1) had been made. There was mild facial involvement and weakness of the neck flexors, wrist extensors, all hand muscles, especially the finger extensors, tibialis anterior, long toe extensors and EDB, with a later proximal involvement including the shoulder girdle and elbow flexors.

This distribution of muscle weakness is similar to that seen in Laing distal myopathy caused by dominant mutations in the myosin heavy chain gene $M Y H 7$ [38,39], and to that seen in recessively inherited distal nebulin myopathy [25], with finger extensors being weaker than the flexors. Better preservation of index finger extension compared with the other fingers, as may be present in recessive distal nebulin myopathy and in Laing distal myopathy, was not observed. These two disorders are the closest differential diagnoses to keep in mind.

Other distal myopathies with foot drop as the presenting feature need to be considered in the differential diagnostic work-up [30].

We describe a novel large pathogenic deletion in $N E B$ in a family with three affected nemaline/cap myopathy patients in three generations. This $\sim 99-106 \mathrm{~kb}$ deletion in $N E B$ covers exons $14-81$ as well as one copy of the $N E B$ triplicate region (exons 82-89). Despite extensive genetic studies using a number of different approaches, no other pathogenic mutations were identified in the known nemaline myopathy-causing genes or in any other plausible candidate genes.

Analysing mRNA from the muscle biopsies of the patients, we were able to demonstrate the presence of both alleles of muscle $N E B$ cDNA. Thus, the mutant allele is being expressed. Importantly, Western blot analysis verified that the mutant protein is being produced, showing two smaller nebulin proteins present in the muscle biopsies of the affected family members.

Based on the evidence above, we conclude that a dominant-negative effect of the smaller-than-normal nebulin protein is the likely cause of the nemaline myopathy in these patients.

In addition to the large deletion in $N E B$, we identified a missense mutation in $M Y H 2$ (p.Ile1515Thr) in the index patient (III-1) and the father (II-3), but not in the mother (II-2). The question was considered whether this alteration might have a modifying effect, perhaps explaining the more severe clinical picture of the index patient compared with the mildly affected mother and grandmother (I-3). However, MYH2 is expressed in fast $2 \mathrm{~A}$ fibres, while the biopsies of the index patient showed mainly type 1 fibres. Since the mother, not carrying the $M Y H 2$ variant, had a similar fibre type distribution and the target muscle tibialis anterior is a muscle with type 1 predominance, an effect of the $M Y H 2$ variant appears unlikely as the explanation. According to the ExAC browser (http://exac.broadinstitute.org/variant/ 12-102040659-G-A), the missense variant p.Val337Ile in $M Y B P C 1$, identified in the index patient and his father, has an allele frequency of 0.005 in the Finnish population. MYBPC1 is predominantly expressed in slow muscle fibres, and a modifying effect of this variant cannot be excluded.

Although the index patient (III-1) underwent two muscle biopsies, typical nemaline bodies were not observed. This might be due to sampling error or it might be age-related, as a number of patients with nemaline myopathy have been described to show no nemaline bodies in their first biopsy or only show them in the electron microscopy sample [40-44]. There are many previous reports of families with congenital myopathy where the histological pictures vary between family members, despite their sharing the same mutation $[45,46]$. Moreover, it is known that cap formation and quantities of nemaline bodies may vary between family members [5,21]. Nemaline rods can be present in focal peripheral zones; similarly cap-like areas can be focal and contain thickened Z-disc material. Rods and cap-like areas may thus be parts of a pathological spectrum $[5,21]$.

To date, we have screened 270 families with nemaline myopathy or related myopathies using the NM-CGH Array, and identified 15 different disease-causing copy number variations in $N E B$ in 31 families. There are still more than 60 families in which we have only identified one $N E B$ mutation, while the second one remains unknown. The present study indicates that in some rare cases a large aberration in $N E B$ can be dominant, while point mutations and small insertions and deletions should still be considered recessive.

\section{Funding}

This study was supported by grants from the Sigrid Jusélius Foundation, the Academy of Finland, the Association 
Francaise contre les Myopathies, Muscular Dystrophy UK, the Finska Läkaresällskapet and the Medicinska understödsföreningen Liv och Hälsa.

\section{Acknowledgements}

We thank Professor Glenn Morris for providing the antibodies against nebulin, and Liina Ahlstén, Helena Luque and Marilotta Turunen for excellent technical assistance.

\section{Supplementary material}

Supplementary material associated with this article can be found, in the online version, at doi:10.1016/j.nmd.2018.12. 007.

\section{References}

[1] Jungbluth H, Wallgren-Pettersson C. Congenital (Structural) myopathies. In: Rimoin DL, Pyeritz RE, Korf BR, editors. Emery \& Rimoin's principles and practice of medical genetics. 6th ed Oxford: Academic Press; 2013. Chapter 127 p. 1-51.

[2] Shy GM, Engel WK, Somers JE, Wanko T. Nemaline myopathy, a new congenital myopathy. Brain 1963;86:793-810.

[3] Dubowitz V, Oldfors A, Sewry CA. Muscle Biopsy; a practical approach. 4th ed. Saunders Elsevier Ltd; 2013.

[4] Piteau SJ, Rossiter JP, Smith RG, MacKenzie JJ. Congenital myopathy with cap-like structures and nemaline rods: case report and literature review. Pediatr Neurol 2014;51:192-7.

[5] Lehtokari VL, Ceuterick-de Groote C, de Jonghe P, Marttila M, Laing NG, Pelin K, et al. Cap disease caused by heterozygous deletion of the beta-tropomyosin gene TPM2. Neuromuscul Disord 2007; 17:433-42.

[6] Lehtokari VL, Pelin K, Herczegfalvi A, Karcagi V, Pouget J, Franques J, et al. Nemaline myopathy caused by mutations in the nebulin gene may present as a distal myopathy. Neuromuscul Disord 2011;21:556-62.

[7] Lehtokari VL, Kiiski K, Sandaradura SA, Laporte J, Repo P, Frey JA, et al. Mutation update: the spectra of nebulin variants and associated myopathies. Hum Mutat 2014;35:1418-26.

[8] Liewluck T, Sorenson EJ, Walkiewicz MA, Rumilla KM, Milone M. Autosomal dominant distal myopathy due to a novel ACTA1 mutation. Neuromuscul Disord 2017;27:742-6.

[9] Laing NG, Wilton SD, Akkari PA, Dorosz S, Boundy K, Kneebone C, et al. A mutation in the alpha tropomyosin gene TPM3 associated with autosomal dominant nemaline myopathy NEM1. Nat Genet 1995;9:75-9.

[10] Pelin K, Hilpela P, Donner K, Sewry C, Akkari PA, Wilton SD, et al. Mutations in the nebulin gene associated with autosomal recessive nemaline myopathy. Proc Natl Acad Sci U.S.A. 1999;96:2305-10.

[11] Nowak KJ, Wattanasirichaigoon D, Goebel HH, Wilce M, Pelin K, Donner K, et al. Mutations in the skeletal muscle alpha-actin gene in patients with actin myopathy and nemaline myopathy. Nat Genet 1999;23:208-12.

[12] Johnston JJ, Kelley RI, Crawford TO, Morton DH, Agarwala R, Koch T, et al. A novel nemaline myopathy in the Amish caused by a mutation in troponin T1. Am J Hum Genet 2000;67:814-21.

[13] Donner K, Ollikainen M, Ridanpaa M, Christen HJ, Goebel HH, de Visser M, et al. Mutations in the beta-tropomyosin (TPM2) gene-a rare cause of nemaline myopathy. Neuromuscul Disord 2002;12:151-8.

[14] Agrawal PB, Greenleaf RS, Tomczak KK, Lehtokari VL, Wallgren-Pettersson C, Wallefeld $\mathrm{W}$, et al. Nemaline myopathy with minicores caused by mutation of the CFL2 gene encoding the skeletal muscle actin-binding protein, cofilin-2. Am J Hum Genet 2007;80:162-7.
[15] Yuen M, Sandaradura SA, Dowling JJ, Kostyukova AS, Moroz N, Quinlan KG, et al. Leiomodin-3 dysfunction results in thin filament disorganization and nemaline myopathy. J Clin Invest 2015;125:456-7.

[16] Miyatake S, Mitsuhashi S, Hayashi YK, Purevjav E, Nishikawa A, Koshimizu E, et al. Biallelic mutations in MYPN, encoding myopalladin, are associated with childhood-onset, slowly progressive nemaline myopathy. Am J Hum Genet 2017;100:169-78.

[17] Sambuughin N, Yau KS, Olive M, Duff RM, Bayarsaikhan M, Lu S, et al. Dominant mutations in KBTBD13, a member of the BTB/Kelch family, cause nemaline myopathy with cores. Am J Hum Genet 2010;87:842-7.

[18] Ravenscroft G, Miyatake S, Lehtokari VL, Todd EJ, Vornanen P, Yau KS, et al. Mutations in KLHL40 are a frequent cause of severe autosomal-recessive nemaline myopathy. Am J Hum Genet 2013;93:6-18.

[19] Gupta VA, Ravenscroft G, Shaheen R, Todd EJ, Swanson LC, Shiina M, et al. Identification of KLHL41 Mutations Implicates BTB-Kelch-Mediated Ubiquitination as an Alternate Pathway to Myofibrillar Disruption in Nemaline Myopathy. Am J Hum Genet 2013;93:1108-17.

[20] Fidzianska A, Badurska B, Ryniewicz B, Dembek I. "Cap disease": new congenital myopathy. Neurology 1981;31:1113-20.

[21] Tajsharghi H, Ohlsson M, Lindberg C, Oldfors A. Congenital myopathy with nemaline rods and cap structures caused by a mutation in the beta-tropomyosin gene (TPM2). Arch Neurol 2007;64:1334-8.

[22] De Paula AM, Franques J, Fernandez C, Monnier N, Lunardi J, Pellissier JF, et al. A TPM3 mutation causing cap myopathy. Neuromuscul Disord 2009;19:685-8.

[23] Hung RM, Yoon G, Hawkins CE, Halliday W, Biggar D, Vajsar J. Cap myopathy caused by a mutation of the skeletal alpha-actin gene ACTA1. Neuromuscul Disord 2010;20:238-40.

[24] Lornage X, Malfatti E, Cheraud C, Schneider R, Biancalana V, Cuisset JM, et al. Recessive MYPN mutations cause cap myopathy with occasional nemaline rods. Ann Neurol 2017;81:467-73.

[25] Wallgren-Pettersson C, Lehtokari VL, Kalimo H, Paetau A, Nuutinen E, Hackman P, et al. Distal myopathy caused by homozygous missense mutations in the nebulin gene. Brain 2007;130:1465-76.

[26] Romero NB, Lehtokari VL, Quijano-Roy S, Monnier N, Claeys KG, Carlier RY, et al. Core-rod myopathy caused by mutations in the nebulin gene. Neurology 2009;73:1159-61.

[27] Scoto M, Cullup T, Cirak S, Yau S, Manzur AY, Feng L, et al. Nebulin (NEB) mutations in a childhood onset distal myopathy with rods and cores uncovered by next generation sequencing. Eur J Hum Genet 2013;21:1249-52.

[28] Abdalla E, Ravenscroft G, Zayed L, Beecroft SJ, Laing NG. Lethal multiple pterygium syndrome: a severe phenotype associated with a novel mutation in the nebulin gene. Neuromuscul Disord 2017;27: $537-541$

[29] Udd B. Distal myopathies-new genetic entities expand diagnostic challenge. Neuromuscul Disord 2012;22:5-12.

[30] Udd B. Distal myopathies. Curr Neurol Neurosci Rep 2014;14:434.

[31] Labeit S, Kolmerer B. The complete primary structure of human nebulin and its correlation to muscle structure. J Mol Biol 1995;248:308-15.

[32] Labeit S, Ottenheijm CA, Granzier H. Nebulin, a major player in muscle health and disease. FASEB J 2011;25:822-9.

[33] Donner K, Sandbacka M, Lehtokari VL, Wallgren-Pettersson C, Pelin K. Complete genomic structure of the human nebulin gene and identification of alternatively spliced transcripts. Eur J Hum Genet 2004; 12:744-51.

[34] Lehtokari VL, Pelin K, Sandbacka M, Ranta S, Donner K, Muntoni F, et al. Identification of 45 novel mutations in the nebulin gene associated with autosomal recessive nemaline myopathy. Hum Mutat 2006;27:946-56

[35] Kiiski K, Lehtokari VL, Loytynoja A, Ahlsten L, Laitila J, Wallgren-Pettersson $\mathrm{C}$, et al. A recurrent copy number variation of the NEB triplicate region: only revealed by the targeted nemaline myopathy CGH array. Eur J Hum Genet 2016;24:574-80. 
[36] Pelin K, Donner K, Holmberg M, Jungbluth H, Muntoni F, Wallgren-Pettersson C. Nebulin mutations in autosomal recessive nemaline myopathy: an update. Neuromuscul Disord 2002;12:680-6.

[37] Kiiski K, Laari L, Lehtokari VL, Lunkka-Hytonen M, Angelini C, Petty R, et al. Targeted array comparative genomic hybridization-a new diagnostic tool for the detection of large copy number variations in nemaline myopathy-causing genes. Neuromuscul Disord 2013;23: 56-65.

[38] Laing NG, Laing BA, Meredith C, Wilton SD, Robbins P, Honeyman K, et al. Autosomal dominant distal myopathy: linkage to chromosome 14. Am J Hum Genet 1995;56:422-7.

[39] Lamont PJ, Udd B, Mastaglia FL, de Visser M, Hedera P, Voit T, et al. Laing early onset distal myopathy: slow myosin defect with variable abnormalities on muscle biopsy. J Neurol Neurosurg Psychiatry 2006;77:208-15.

[40] Couchot J, Schmauch MA, Caulet T, Pennaforte F, Fandre M. Proceedings: rod myopathy ("nemaline myopathy"). A new fatal case. Arch Fr Pediatr 1975;32:297-8.
[41] Karpati G, Carpenter S, Andermann F. A new concept of childhood nemaline myopathy. Arch Neurol 1971;24:291-304.

[42] Nienhuis AW, Coleman RF, Brown WJ, Munsat TL, Pearson CM. Nemaline myopathy. A histopathologic and histochemical study. Am J Clin Pathol 1967;48:1-13.

[43] Sreter FA, Astrom KE, Romanul FC, Young RR, Jones HR Jr. Characteristics of myosin in nemaline myopathy. J Neurol Sci 1976;27:99-116.

[44] Wallgren-Pettersson C, Rapola J, Donner M. Pathology of congenital nemaline myopathy. A follow-up study. J Neurol Sci 1988;83:243-57.

[45] Afifi AK, Smith JW, Zellweger H. Congenital nonprogressive myopathy. Central core disease and nemaline myopathy in one family. Neurology 1965;15:371-81.

[46] Casado JL, Arenas C, Segura D, Chinchon I, Gonzalez R, Bautista J. Congenital myopathy with cores and nemaline rods in one family. Neurologia 1995;10:145-8. 